\title{
CONVERGENCE IN VARIATION FOR THE MULTIDIMENSIONAL GENERALIZED SAMPLING SERIES AND APPLICATIONS TO SMOOTHING FOR DIGITAL IMAGE PROCESSING
}

\author{
Laura Angeloni, Danilo Costarelli and Gianluca Vinti \\ University of Perugia, Department of Mathematics and Computer Science \\ Via Vanvitelli 1, 06123, Perugia, Italy; laura.angeloni@unipg.it \\ University of Perugia, Department of Mathematics and Computer Science \\ Via Vanvitelli 1, 06123, Perugia, Italy; danilo.costarelli@unipg.it \\ University of Perugia, Department of Mathematics and Computer Science \\ Via Vanvitelli 1, 06123, Perugia, Italy; gianluca.vinti@unipg.it
}

\begin{abstract}
In this paper we study the problem of the convergence in variation for the generalized sampling series based upon averaged-type kernels in the multidimensional setting. As a crucial tool, we introduce a family of operators of sampling-Kantorovich type for which we prove convergence in $L^{p}$ on a subspace of $L^{p}\left(\mathbf{R}^{N}\right)$ : therefore we obtain the convergence in variation for the multidimensional generalized sampling series by means of a relation between the partial derivatives of such operators acting on an absolutely continuous function $f$ and the sampling-Kantorovich type operators acting on the partial derivatives of $f$. Applications to digital image processing are also furnished.
\end{abstract}

\section{Introduction}

In this paper we present approximation results in BV-spaces for the generalized sampling series in the multidimensional frame.

The above sampling series, defined as

$$
\left(S_{w} f\right)(t):=\sum_{k \in \mathbf{Z}} f\left(\frac{k}{w}\right) \chi(w t-k), \quad t \in \mathbf{R}, w>0,
$$

has been introduced by Butzer (see, e.g., [24]) and revealed to be very interesting for both the theoretical and applicative aspects. Indeed, several approximation results have been obtained in the last forty years with respect to different notions of convergence, such as uniform, $L^{p}$, and modular convergence $[18,37,16]$. Moreover, they have many important applications to Signal Theory since they provide an approximate sampling formula, which allows to reconstruct not necessarily band-limited signals. By its multivariate generalization, that is,

$$
\left(S_{w} f\right)(\mathrm{t}):=\sum_{\mathbf{k} \in \mathbf{Z}^{N}} f\left(\frac{\mathbf{k}}{w}\right) \chi(w \mathbf{t}-\mathbf{k}), \quad \mathbf{t} \in \mathbf{R}^{N}, w>0,
$$

several problems of image processing can be treated (see, e.g., [17]).

A natural setting to study some issues involving digital images is furnished by the spaces of functions of bounded variation [36, 44, 47]. In this direction, it is interesting

https://doi.org/10.5186/aasfm.2020.4532

2010 Mathematics Subject Classification: Primary 41A30, 41A05.

Key words: convergence in variation, multidimensional generalized sampling series, samplingKantorovich operators, variation diminishing type property, smoothing in digital image processing. 
to have results about estimates and convergence in variation for the above discrete operators. In the case of functions of one-variable, the problem has been faced in [6] for the operators (I) based upon averaged type kernels.

Here, also with the aim to consider applicative aspects, we deal with the multivariate case, namely we study results about approximation in variation by means of the operators (II) based upon multidimensional product kernels of averaged type, namely

$$
\bar{\chi}_{m}(\mathrm{t}):=\prod_{i=1}^{N} \bar{\chi}_{i, m}\left(t_{i}\right)
$$

where

$$
\bar{\chi}_{i, m}(t):=\frac{1}{m} \int_{-\frac{m}{2}}^{\frac{m}{2}} \chi_{i}(t+v) d v
$$

for some $m \in \mathbf{N}$, and $\chi_{i}: \mathbf{R} \longrightarrow \mathbf{R}$ are suitable one-dimensional kernels. We will use the multidimensional generalization of the classical Jordan variation introduced by Tonelli (see, e.g., [45]) and later generalized by Radó [40] and Vinti [48] to the case of functions of $N$-variables.

In particular, we establish a convergence theorem (Theorem 1).

Due to form of the above discrete sampling type operators, to obtain results about approximation in variation is a very delicate problem. The strategy we propose is to introduce a new family of Kantorovich type operators defined as

$$
\left(K_{w, j} f\right)(\mathrm{t}):=\sum_{\mathbf{k} \in \mathbf{Z}^{N}}\left[w \int_{\frac{k_{j}}{w}}^{\frac{k_{j}+1}{w}} f\left(\frac{k_{1}}{w}, \ldots, u \ldots, \frac{k_{N}}{w}\right) d u\right] \chi(w \mathbf{t}-\mathbf{k}),
$$

$\mathrm{t} \in \mathbf{R}^{N}, w>0, j=1, \ldots, N$, for which we establish $L^{p}$-convergence in a subspace of $L^{p}\left(\mathbf{R}^{N}\right)$ (Theorem 4 ), therefore obtaining a convergence result for the new class of operators (III). We notice that the above result is obtained through the use of the $\tau$-modulus of continuity (see $[34,33,42]$ ) which seems to be the most suitable approach in this setting.

The crucial point in order to reach the convergence in variation is to find a link between the two classes of operators: to this aim we prove (Proposition 2) a relation between the gradient of the multivariate generalized sampling series of a function $f$ and the family of Kantorovich-type discrete operators acting on the partial derivatives of $f$.

Some of the results of the present paper may have an applicative interpretation: in particular, we prove a variation diminishing type property (Proposition 5) ensuring that, in case of some step-type functions, the variation of the generalized sampling sampling series (II) is controlled by the variation of the function on which they act, and this can be viewed as a smoothing procedure. Indeed, if the function $f$ is a gray scale digital image, the action of the operators and the consequent variation diminishing type property corresponds, in some sense, to reduce the jumps of gray levels with respect to the original image, hence producing a smoothing effect. In Section 6 we discuss such digital image processing applications in details, furnishing some numerical examples.

\section{Notations and preliminaries}

Our results will be set in the frame of the space of multivariate functions of bounded variation: in particular we will consider the concept of variation introduced 
by Tonelli [45] for two variables, extended to the general case of $\mathbf{R}^{N}$ by Radó and Vinti $[40,48]$. Here we will recall it.

For a function $f: \mathbf{R}^{N} \rightarrow \mathbf{R}$ and $\mathbf{x}=\left(x_{1}, \ldots, x_{N}\right) \in \mathbf{R}^{N}$, we will use the notation

$$
\mathrm{x}_{j}^{\prime}=\left(x_{1}, \ldots, x_{j-1}, x_{j+1}, \ldots, x_{N}\right) \in \mathbf{R}^{N-1}, \quad \mathbf{x}=\left(\mathrm{x}_{j}^{\prime}, x_{j}\right), \quad f(\mathrm{x})=f\left(\mathrm{x}_{j}^{\prime}, x_{j}\right),
$$

if we are interested in the $j$-th coordinate of $\mathrm{x}, j=1, \ldots, N$. Moreover, given an $N$-dimensional interval $I=\prod_{i=1}^{N}\left[a_{i}, b_{i}\right]$, by $I_{j}^{\prime}=\left[\mathrm{a}_{j}^{\prime}, \mathrm{b}_{j}^{\prime}\right]$ we will denote the $(N-1)$-dimensional interval obtained deleting by $I$ the $j$-th coordinate, i.e.,

$$
I=\left[\mathrm{a}_{j}^{\prime}, \mathrm{b}_{j}^{\prime}\right] \times\left[a_{j}, b_{j}\right], \quad j=1, \ldots, N .
$$

Given a vector $\mathbf{x} \in \mathbf{R}^{N}$ and $\alpha \in \mathbf{R}$, we will use the usual notation for products and quotients, i.e., $\alpha \mathrm{x}=\left(\alpha x_{1}, \ldots, \alpha x_{N}\right)$ and, for $\alpha \neq 0, \frac{\mathbf{x}}{\alpha}=\left(\frac{x_{1}}{\alpha}, \ldots, \frac{x_{N}}{\alpha}\right)$.

By $R_{\text {loc }}\left(\mathbf{R}^{N}\right)$ we will denote the space of locally Riemann integrable functions on $\mathbf{R}^{N}$, while $M\left(\mathbf{R}^{N}\right)$ will denote the space of all the measurable and bounded functions $f: \mathbf{R}^{N} \rightarrow \mathbf{R}$.

Definition 1. A function $f \in M\left(\mathbf{R}^{N}\right)$ is said to be of bounded variation $(f \in$ $B V\left(\mathbf{R}^{N}\right)$ ) if $V_{\mathbf{R}}\left[f\left(\mathrm{x}_{j}^{\prime}, \cdot\right)\right]$ (the usual Jordan one-dimensional variation of the $j$-th section of $f)$ is finite for a.e. $\mathbf{x}_{j}^{\prime} \in \mathbf{R}^{N-1}$ and

for every $j=1, \ldots, N$.

$$
\int_{\mathbf{R}^{N-1}} V_{\mathbf{R}}\left[f\left(\mathrm{x}_{j}^{\prime}, \cdot\right)\right] d \mathrm{x}_{j}^{\prime}<+\infty
$$

For more details about $B V$-spaces, see, e.g., $[9,5,10,11,4,13]$.

Let us now recall how to compute the multidimensional Tonelli variation. The first step is to consider, for $I=\prod_{i=1}^{N}\left[a_{i}, b_{i}\right]$ and $j=1, \ldots, N$ the $(N-1)$-dimensional integrals

$$
\Phi_{j}(f, I):=\int_{\left[\mathrm{a}_{j}^{\prime}, \mathrm{b}_{j}^{\prime}\right]} V_{\left[a_{j}, b_{j}\right]}\left[f\left(\mathrm{x}_{j}^{\prime}, \cdot\right)\right] d \mathrm{x}_{j}^{\prime},
$$

where $V_{\left[a_{j}, b_{j}\right]}\left[f\left(\mathrm{x}_{j}^{\prime}, \cdot\right)\right]$ is the usual one-dimensional (Jordan) variation of the $j$-th section of $f$. Let now $\Phi(f, I)$ be the Euclidean norm of the vector $\left(\Phi_{1}(f, I), \ldots, \Phi_{N}(f, I)\right)$, namely

$$
\Phi(f, I):=\left\{\sum_{j=1}^{N} \Phi_{j}^{2}(f, I)\right\}^{\frac{1}{2}},
$$

where $\Phi(f, I)=+\infty$ if $\Phi_{j}(f, I)=+\infty$ for some $j=1, \ldots, N$.

Then the variation of $f$ on $I \subset \mathbf{R}^{N}$ is defined as

$$
V_{I}[f]:=\sup \sum_{k=1}^{m} \Phi\left(f, J_{k}\right),
$$

where the supremum is taken over all the finite families of $N$-dimensional intervals $\left\{J_{1}, \ldots, J_{m}\right\}$ which form partitions of $I$.

Passing to the supremum over all the intervals $I \subset \mathbf{R}^{N}$, we obtain the variation of $f$ over the whole $\mathbf{R}^{N}$, i.e.,

$$
V[f]:=\sup _{I \subset \mathbf{R}^{N}} V_{I}[f] .
$$

It is well known that, for every $f \in B V\left(\mathbf{R}^{N}\right), \nabla f$ exists a.e. in $\mathbf{R}^{N}$ and $\frac{\partial f}{\partial x_{j}} \in L^{1}\left(\mathbf{R}^{N}\right)$, for every $j=1, \ldots, N$ (see e.g. [40, 48]).

We now recall the notion of absolute continuity in the sense of Tonelli. 
Definition 2. A function $f: \mathbf{R}^{N} \rightarrow \mathbf{R}$ is locally absolutely continuous in the sense of Tonelli $\left(f \in A C_{\text {loc }}\left(\mathbf{R}^{N}\right)\right)$ if, for every interval $I=\prod_{i=1}^{N}\left[a_{i}, b_{i}\right]$ and for every $j=1,2, \ldots, N$, the $j$-th section of $f, f\left(\mathrm{x}_{j}^{\prime}, \cdot\right):\left[a_{j}, b_{j}\right] \rightarrow \mathbf{R}$ is absolutely continuous for almost every $\mathrm{x}_{j}^{\prime} \in\left[\mathrm{a}_{j}^{\prime}, \mathrm{b}_{j}^{\prime}\right]$.

It is a well known result that, if $f \in B V\left(\mathbf{R}^{N}\right) \cap A C_{\text {loc }}\left(\mathbf{R}^{N}\right)$, then

$$
V[f]=\int_{\mathbf{R}^{N}}|\nabla f(\mathrm{x})| d \mathrm{x}
$$

(see $[40,48,35,12]$ ), that is, an integral representation for the variation of $f$ holds.

We will therefore denote by $A C\left(\mathbf{R}^{N}\right)$ the space of all the functions $f \in B V\left(\mathbf{R}^{N}\right) \cap$ $A C_{\text {loc }}\left(\mathbf{R}^{N}\right)$ (absolutely continuous functions).

We will study approximation results for the multivariate generalized sampling series, namely a family of discrete operators defined as

$$
\left(S_{w} f\right)(\mathrm{t}):=\sum_{\mathbf{k} \in \mathbf{Z}^{N}} f\left(\frac{\mathbf{k}}{w}\right) \chi(w \mathrm{t}-\mathbf{k}), \quad \mathbf{t} \in \mathbf{R}^{N}, w>0:
$$

such operators are the multidimensional version of the generalized sampling series (see, e.g., $[21,22,17])$.

Here $\chi$ is a kernel, that is, a function $\chi: \mathbf{R}^{N} \rightarrow \mathbf{R}$ that satisfies the following assumptions:

$\left(\chi_{1}\right) \chi \in L^{1}\left(\mathbf{R}^{N}\right)$ is such that $\sum_{\mathrm{k} \in \mathbf{Z}^{N}} \chi(\mathrm{u}-\mathrm{k})=1$, for every $\mathrm{u} \in \mathbf{R}^{N}$;

$\left(\chi_{2}\right) A_{\chi}:=\sup _{\mathrm{u} \in \mathbf{R}^{N}} \sum_{\mathbf{k} \in \mathbf{Z}^{N}}|\chi(\mathrm{u}-\mathrm{k})|<+\infty$, where the convergence of the series is uniform on the compact sets of $\mathbf{R}^{N}$.

The above assumptions are quite standard working with discrete families of operators: see, e.g., [21]. We point out that the operators $\left(S_{w} f\right)_{w>0}$ are well-defined, for example, for every $f \in B V\left(\mathbf{R}^{N}\right)$ : indeed in this case $f$ is bounded and since $|f(\mathrm{t})| \leq M$, for some $M>0$ and for every $\mathrm{t} \in \mathbf{R}^{N}$, we have

$$
\left|\left(S_{w} f\right)(\mathrm{t})\right| \leq M \sum_{\mathbf{k} \in \mathbf{Z}^{N}}|\chi(w \mathrm{t}-\mathbf{k})| \leq M A_{\chi}
$$

by $\left(\chi_{2}\right)$.

In particular, in the present paper we will study the convergence in variation for the multivariate generalized sampling series with product kernels of averaged type, that is kernels of the form

$$
\bar{\chi}_{m}(\mathrm{t}):=\prod_{i=1}^{N} \bar{\chi}_{i, m}\left(t_{i}\right)
$$

where

$$
\bar{\chi}_{i, m}(t):=\frac{1}{m} \int_{-\frac{m}{2}}^{\frac{m}{2}} \chi_{i}(t+v) d v,
$$

for some $m \in \mathbf{N}$, and $\chi_{i}: \mathbf{R} \longrightarrow \mathbf{R}$ is a (one-dimensional) kernel for every $i=$ $1, \ldots, N$ (i.e., satisfying $\left(\chi_{1}\right)$ and $\left(\chi_{2}\right)$ with $N=1$ ).

Notice that it is easy to see that $\bar{\chi}_{m}$ is a kernel itself and moreover, by the Fubini-Tonelli theorem,

$$
\begin{aligned}
\left\|\bar{\chi}_{i, m}\right\|_{1} & =\int_{\mathbf{R}}\left|\frac{1}{m} \int_{-\frac{m}{2}}^{\frac{m}{2}} \chi_{i}(t+v) d v\right| d t \leq \frac{1}{m} \int_{-\frac{m}{2}}^{\frac{m}{2}} \int_{\mathbf{R}}\left|\chi_{i}(t+v)\right| d t d v \\
& =\left\|\chi_{i}\right\|_{1}
\end{aligned}
$$


and, by this,

$$
\left\|\bar{\chi}_{m}\right\|_{1} \leq \prod_{i=1}^{N}\left\|\chi_{i}\right\|_{1}
$$

We point out that is it easy to give examples of product kernels of averaged type (see Section 6) to which our results can be applied.

The corresponding multivariate generalized sampling series (associated to the averaged product kernel $\bar{\chi}_{m}$ ) will be denoted as

$$
\left(\bar{S}_{w}^{m} f\right)(\mathrm{t}):=\sum_{\mathbf{k} \in \mathbf{Z}^{N}} f\left(\frac{\mathbf{k}}{w}\right) \bar{\chi}_{m}(w \mathbf{t}-\mathbf{k}), \quad \mathbf{t} \in \mathbf{R}^{N}, w>0 .
$$

It is easy to see that $\bar{\chi}_{m}$ is differentiable and, taking into account that, obviously,

$$
\frac{\partial \bar{\chi}_{m}}{\partial t_{j}}(\mathrm{t})=\frac{1}{m} \prod_{i \neq j} \bar{\chi}_{i, m}\left(t_{i}\right)\left[\chi_{j}\left(t_{j}+\frac{m}{2}\right)-\chi_{j}\left(t_{j}-\frac{m}{2}\right)\right], \quad \mathbf{t} \in \mathbf{R}^{N}
$$

it is possible to write, for every $j=1, \ldots, N, \mathbf{t} \in \mathbf{R}^{N}, w>0$,

$$
\begin{aligned}
\frac{\partial \bar{S}_{w}^{m} f}{\partial t_{j}}(\mathrm{t})= & \frac{w}{m} \sum_{\mathbf{k} \in \mathbf{Z}^{N}} f\left(\frac{\mathbf{k}}{w}\right) \prod_{i \neq j} \bar{\chi}_{i, m}\left(w t_{i}-k_{i}\right) \\
& \cdot\left[\chi_{j}\left(w t_{j}-k_{j}+\frac{m}{2}\right)-\chi_{j}\left(w t_{j}-k_{j}-\frac{m}{2}\right)\right] .
\end{aligned}
$$

Notice that, since $f$ is bounded, $\frac{\partial \bar{S}_{w}^{m} f}{\partial t_{j}}(\mathrm{t})$ exists for every $\mathbf{t} \in \mathbf{R}^{N}$ since

$$
\begin{aligned}
\left|\frac{\partial \bar{S}_{w}^{m} f}{\partial t_{j}}(\mathrm{t})\right| \leq & \frac{w}{m} M \sum_{\mathbf{k} \in \mathbf{Z}^{N}} \prod_{i \neq j}\left|\bar{\chi}_{i, m}\left(w t_{i}-k_{i}\right)\right| \\
& \cdot\left[\left|\chi_{j}\left(w t_{j}-k_{j}+\frac{m}{2}\right)\right|+\left|\chi_{j}\left(w t_{j}-k_{j}-\frac{m}{2}\right)\right|\right] \\
\leq & \frac{2 w}{m} M \prod_{i=1}^{N} A_{\chi_{i}},
\end{aligned}
$$

again by $\left(\chi_{2}\right)$ for each one-dimensional kernel $\chi_{i}$.

One of the main ideas of this paper is to establish a relation between the gradient of the multivariate generalized sampling series of a function $f$ and a family of Kantorovich-type discrete operators, that we now introduce, acting on the partial derivatives of $f$. This generalizes the analogous result, in the one-dimensional case, obtained in [6], similarly to what happens between the Bernstein polynomials and their Kantorovich version (see, e.g., [1]).

We therefore introduce the following family of multidimensional discrete operators of sampling-Kantorovich type:

$$
\left(K_{w, j} f\right)(\mathrm{t}):=\sum_{\mathbf{k} \in \mathbf{Z}^{N}}\left[w \int_{\frac{k_{j}}{w}}^{\frac{k_{j}+1}{w}} f\left(\frac{k_{1}}{w}, \ldots, u \ldots, \frac{k_{N}}{w}\right) d u\right] \chi(w \mathbf{t}-\mathbf{k}), \quad \mathbf{t} \in \mathbf{R}^{N}, w>0,
$$

$j=1, \ldots, N$. Notice that, similarly to the case of $\left(S_{w} f\right)_{w>0}$, it is easy to see that the operators $\left(K_{w, j} f\right)_{w>0}, j=1, \ldots, N$, are well-defined if, for example $f \in B V\left(\mathbf{R}^{N}\right)$, since $f$ is in particular bounded. 


\section{Results for the multidimensional generalized sampling series}

We will first prove that the operators $\left(\bar{S}_{w}^{m} f\right)_{w>0} \operatorname{map} B V\left(\mathbf{R}^{N}\right)$ into $A C_{l o c}\left(\mathbf{R}^{N}\right)$.

Proposition 1. Let $f \in B V\left(\mathbf{R}^{N}\right)$. Then $\bar{S}_{w}^{m} f \in A C_{l o c}\left(\mathbf{R}^{N}\right)$, for every $w>0$, $m \in \mathbf{N}$, and $V\left[\bar{S}_{w}^{m} f\right]=\int_{\mathbf{R}^{N}}\left|\nabla\left(\bar{S}_{w}^{m} f\right)(\mathrm{t})\right| d \mathrm{t}$.

Proof. Let us fix $w>0$ and $m \in \mathbf{N}$. By (3) and (4), the partial derivatives of $\bar{S}_{w}^{m} f$ exist everywhere and are bounded: this implies that the sections of the function $f$ are locally absolutely continuous, namely $f \in A C_{\text {loc }}\left(\mathbf{R}^{N}\right)$. Now, since $\bar{S}_{w}^{m} f \in A C_{\text {loc }}(\mathbf{R})$, for every $I=\prod_{i=1}^{N}\left[a_{i}, b_{i}\right] \subset \mathbf{R}^{N}$ there holds $V_{I}\left[\bar{S}_{w}^{m} f\right]=\int_{I}\left|\nabla\left(\bar{S}_{w}^{m} f\right)(\mathrm{t})\right| d \mathrm{t}$, and so $V\left[\bar{S}_{w}^{m} f\right]=\sup _{I \subset \mathbf{R}^{N}} V_{I}\left[\bar{S}_{w}^{m} f\right]=\sup _{I \subset \mathbf{R}^{N}} \int_{I}\left|\nabla\left(\bar{S}_{w}^{m} f\right)(\mathrm{t})\right| d \mathrm{t}=\int_{\mathbf{R}^{N}}\left|\nabla\left(\bar{S}_{w}^{m} f\right)(\mathrm{t})\right| d \mathrm{t}$.

We will now give the result that establishes a relation between the partial derivatives of the multidimensional sampling series and the multidimensional samplingKantorovich type operators acting on the partial derivatives of the function.

Proposition 2. If $f \in A C\left(\mathbf{R}^{N}\right)$, then for every $\mathrm{t} \in \mathbf{R}^{N}, j=1, \ldots, N$,

$$
\frac{\partial \bar{S}_{w}^{m} f}{\partial t_{j}}(\mathrm{t})=\frac{1}{m} \sum_{i=1}^{m}\left(K_{w, j} \frac{\partial f}{\partial t_{j}}\right)\left(\mathrm{t}_{j}^{\prime}, t_{j}-\frac{m-2(i-1)}{2 w}\right)
$$

$w>0, m \in \mathbf{N}$.

Proof. By (3), since $f$ is locally absolutely continuous, we have that

$$
\begin{aligned}
\frac{\partial \bar{S}_{w}^{m} f}{\partial t_{j}}(\mathrm{t})= & \frac{w}{m} \sum_{\mathbf{k} \in \mathbf{Z}^{N}} f\left(\frac{\mathbf{k}}{w}\right) \prod_{i \neq j} \bar{\chi}_{i, m}\left(w t_{i}-k_{i}\right) \\
& \cdot\left[\chi_{j}\left(w t_{j}-k_{j}+\frac{m}{2}\right)-\chi_{j}\left(w t_{j}-k_{j}-\frac{m}{2}\right)\right] \\
= & \frac{w}{m} \sum_{\mathbf{k} \in \mathbf{Z}^{N}}\left[\int_{0}^{\frac{k_{j}}{w}} \frac{\partial f}{\partial t_{j}}\left(\frac{\mathbf{k}_{j}^{\prime}}{w}, u\right) d u+f\left(\frac{\mathbf{k}_{j}^{\prime}}{w}, 0\right)\right] \\
& \cdot \prod_{i \neq j} \bar{\chi}_{i, m}\left(w t_{i}-k_{i}\right)\left[\chi_{j}\left(w t_{j}-k_{j}+\frac{m}{2}\right)-\chi_{j}\left(w t_{j}-k_{j}-\frac{m}{2}\right)\right] \\
= & \frac{w}{m} \sum_{\mathbf{k} \in \mathbf{Z}^{N}}\left[\int_{0}^{\frac{k_{j}}{w}} \frac{\partial f}{\partial t_{j}}\left(\frac{\mathbf{k}_{j}^{\prime}}{w}, u\right) d u+f\left(\frac{\mathbf{k}_{j}^{\prime}}{w}, 0\right)\right] \\
& \cdot \prod_{i \neq j} \bar{\chi}_{i, m}\left(w t_{i}-k_{i}\right) \chi_{j}\left(w t_{j}-k_{j}+\frac{m}{2}\right) \\
& -\frac{w}{m} \sum_{\mathbf{k} \in \mathbf{Z}^{N}}\left[\int_{0}^{\frac{k_{j}}{w}} \frac{\partial f}{\partial t_{j}}\left(\frac{\mathbf{k}_{j}^{\prime}}{w}, u\right) d u+f\left(\frac{\mathbf{k}_{j}^{\prime}}{w}, 0\right)\right] \\
& \cdot \prod_{i \neq j} \bar{\chi}_{i, m}\left(w t_{i}-k_{i}\right) \chi_{j}\left(w t_{j}-k_{j}-\frac{m}{2}\right) .
\end{aligned}
$$


Now putting in the first series $\widetilde{k}_{j}=k_{j}-m$ and $\widetilde{k}_{i}=k_{i}$ for every $i \neq j$, there holds

$$
\begin{aligned}
& \frac{\partial \bar{S}_{w}^{m} f}{\partial t_{j}}(\mathrm{t})= \frac{w}{m} \sum_{\widetilde{\mathbf{k}} \in \mathbf{Z}^{N}}\left[\int_{0}^{\frac{\widetilde{k}_{j}+m}{w}} \frac{\partial f}{\partial t_{j}}\left(\frac{\widetilde{\mathbf{k}}_{j}^{\prime}}{w}, u\right) d u+f\left(\frac{\widetilde{\mathbf{k}}_{j}^{\prime}}{w}, 0\right)\right] \\
& \cdot \prod_{i \neq j} \bar{\chi}_{i, m}\left(w t_{i}-\widetilde{k}_{i}\right) \chi_{j}\left(w t_{j}-\widetilde{k}_{j}-\frac{m}{2}\right) \\
&-\frac{w}{m} \sum_{\mathbf{k} \in \mathbf{Z}^{N}}\left[\int_{0}^{\frac{k_{j}}{w}} \frac{\partial f}{\partial t_{j}}\left(\frac{\mathbf{k}_{j}^{\prime}}{w}, u\right) d u+f\left(\frac{\mathbf{k}_{j}^{\prime}}{w}, 0\right)\right] \\
&=\frac{w}{m} \sum_{\mathbf{k} \in \mathbf{Z}^{N}} \int_{\frac{k_{j}}{w}}^{\frac{k_{j}+m}{w}} \frac{\partial f}{\partial t_{j}}\left(\frac{\mathbf{k}_{j}^{\prime}}{w}, u\right) d u \prod_{i \neq j} \bar{\chi}_{i, m}\left(w t_{i}-k_{i}\right) \chi_{j}\left(w t_{j}-k_{j}-\frac{m}{2}\right) \\
&=\frac{w}{m} \sum_{\mathbf{k} \in \mathbf{Z}^{N}}\left(\sum_{i=1}^{m} \int_{\frac{k_{j}+i-1}{w}}^{\frac{k_{j}+i}{w}}\right) \frac{\partial f}{\partial t_{j}}\left(\frac{\mathbf{k}_{j}^{\prime}}{w}, u\right) d u \prod_{i \neq j} \bar{\chi}_{i, m}\left(w t_{i}-k_{i}\right) \chi_{j}\left(w t_{j}-k_{j}-\frac{m}{2}\right) \\
&=\frac{1}{m}\left\{\left(K_{w, j} \frac{\partial f}{\partial t_{j}}\right)\left(\mathrm{t}_{j}^{\prime}, t_{j}-\frac{m}{2 w}\right)+\ldots+\left(K_{w, j} \frac{\partial f}{\partial t_{j}}\right)\left(\mathrm{t}_{j}^{\prime}, t_{j}-\frac{m-2(m-1)}{2 w}\right)\right\} \\
&=\frac{1}{m} \sum_{i=1}^{m}\left(K_{w, j} \frac{\partial f}{\partial t_{j}}\right)\left(\mathrm{t}_{j}^{\prime}, t_{j}-\frac{m-2(i-1)}{2 w}\right) .
\end{aligned}
$$

\section{Convergence in $L^{p}$ for the multidimensional sampling-Kantorovich type operators}

We now study the problem of the convergence in $L^{p}$ for the multidimensional sampling-Kantorovich type operators that we introduced. Such result will be also fundamental in order to prove the convergence in variation for $\left(\bar{S}_{w} f\right)_{w>0}$.

We recall that convergence in $L^{p}$ holds for the multidimensional generalized sampling series $\left(S_{w} f\right)_{w>0}$ (see [17]) assuming that $f$ belongs to a suitable subspace of $L^{p}\left(\mathbf{R}^{N}\right)$, namely $\Lambda^{p}$. Since the definition of our operators $\left(K_{w, j} f\right)_{w>0}$ is very close to that one of $\left(S_{w} f\right)_{w>0}$, it is natural to expect that the $L^{p}$-convergence holds within the same subspace, that is what we will now prove.

Let us recall the definition of $\Lambda^{p}$ (see [17]).

Let us consider an admissible partition over the $i$-th axis, i.e., $\Sigma_{i}:=\left(x_{i, j_{i}}\right)_{j_{i} \in \mathbf{Z}}$ such that

$$
0<\underline{\Delta}:=\min _{i=1, \ldots, N} \inf _{j_{i} \in \mathbf{Z}}\left(x_{i, j_{i}}-x_{i, j_{i}-1}\right) \leq \max _{i=1, \ldots, N} \sup _{j_{i} \in \mathbf{Z}}\left(x_{i, j_{i}}-x_{i, j_{i}-1}\right)=: \bar{\Delta}<+\infty .
$$

We say that $\Sigma=\left(\mathrm{x}_{\mathrm{j}}\right)_{\mathrm{j} \in \mathbf{Z}^{N}} \subset \mathbf{R}^{N}, \mathrm{x}_{\mathrm{j}}=\left(x_{1, j_{1}}, \ldots, x_{N, j_{N}}\right), \mathrm{j}=\left(j_{1}, \ldots, j_{N}\right) \in \mathbf{Z}^{N}$, is an admissible sequence if it is the cartesian product of admissible partitions $\Sigma_{i}=$ $\left(x_{i, j_{i}}\right)_{j_{i} \in \mathbf{Z}}$.

For a fixed admissible sequence $\Sigma$, the $l^{p}(\Sigma)$-norm of a function $f: \mathbf{R}^{N} \rightarrow \mathbf{R}$ is defined as

$$
\|f\|_{l^{p}(\Sigma)}:=\left\{\sum_{\mathbf{j} \in \mathbf{Z}^{N}} \sup _{\mathbf{x} \in Q_{\mathbf{j}}}|f(\mathrm{x})|^{p} \Delta_{\mathrm{j}}\right\}^{\frac{1}{p}}, \quad 1 \leq p<+\infty
$$


where $Q_{\mathrm{j}}=\prod_{i=1}^{N}\left[x_{i, j_{i}-1}-x_{i, j_{i}}\right.$ [ and $\Delta_{\mathrm{j}}:=\prod_{i=1}^{N}\left(x_{i, j_{i}}-x_{i, j_{i}-1}\right)$ is the volume of $Q_{\mathrm{j}}$. With such notations the subspace $\Lambda^{p}$ is defined as

$$
\Lambda^{p}:=\left\{f \in M\left(\mathbf{R}^{N}\right):\|f\|_{l^{p}(\Sigma)}<+\infty, \text { for every admissible sequence } \Sigma\right\} .
$$

In [17] it is proved that $\Lambda^{p}$ is a proper linear subspace of $L^{p}\left(\mathbf{R}^{N}\right)$, together with other properties concerning such space. In particular, we recall the following important result of convergence in $L^{p}$ for the $\tau$-modulus of smoothness (see $[34,33,42]$ )

$$
\tau_{r}\left(f ; \delta, M\left(\mathbf{R}^{N}\right)\right)_{p}:=\left\|\omega_{r}\left(f ; \cdot, \delta, M\left(\mathbf{R}^{N}\right)\right)\right\|_{p},
$$

where

$$
\omega_{r}\left(f ; \mathrm{x}, \delta, M\left(\mathbf{R}^{N}\right)\right):=\sup \left\{\left|\Delta_{\mathrm{h}}^{r} f(\mathrm{t})\right|: \mathrm{t}, \mathrm{t}+\mathrm{h} r \in \prod_{i=1}^{N}\left[x_{i}-\frac{\delta r}{2}, x_{i}+\frac{\delta r}{2}\right]\right\},
$$

$\delta>0$, and $\Delta_{\mathrm{h}}^{r} f(\mathrm{t}):=\sum_{j=0}^{r}(-1)^{r+j}\left(\begin{array}{l}r \\ j\end{array}\right) f(\mathrm{t}+j \mathrm{~h})$ denotes the differences of order $r$ at $\mathrm{t} \in \mathbf{R}^{N}$ with increment $\mathrm{h} \in \mathbf{R}^{N}$. then

Proposition 3. [17, Proposition 7] If $f \in \Lambda^{p} \cap R_{\mathrm{loc}}\left(\mathbf{R}^{N}\right), 1 \leq p<+\infty, r \in \mathbf{N}$,

$$
\lim _{\delta \rightarrow 0^{+}} \tau_{r}\left(f ; \delta, M\left(\mathbf{R}^{N}\right)\right)_{p}=0 .
$$

We point out that, of course, $\Lambda^{p}$ is a non trivial subspace since it contains, for example, all the functions in $M\left(\mathbf{R}^{N}\right)$ with compact support.

We are now ready to state the result of convergence in $L^{p}$ for the multidimensional sampling-Kantorovich type operators.

Let us now assume that $\chi$ is a kernel with compact support, i.e., $\chi(\mathrm{t})=0$ if $\mathrm{t} \notin[-T, T]^{N}, T>0$, not necessarily of product type, namely $\chi$ satisfies the assumption:

$(\chi)$ there exists $T>0$ such that $\chi(\mathrm{t})=0$ for $\mathrm{t} \notin[-T, T]^{N}$ and $\sum_{\mathrm{k} \in \mathbf{Z}^{N}} \chi(\mathrm{u}-\mathrm{k})=$ 1 , for every $\mathrm{u} \in \mathbf{R}^{N}$.

We point out that, since $\chi$ has compact support, obviously $\chi \in L^{1}\left(\mathbf{R}^{N}\right)$ (and therefore $\left(\chi_{1}\right)$ holds) and satisfies the condition $\left(\chi_{2}\right)$ since $\chi$ is bounded and the series reduces to a finite sum.

Proposition 4. Let $f \in \Lambda^{p} \cap R_{\text {loc }}\left(\mathbf{R}^{N}\right), 1 \leq p<+\infty$. Then, for every $j=$ $1, \ldots, N$

$$
\lim _{w \rightarrow+\infty}\left\|K_{w, j} f-f\right\|_{p}=0 .
$$

Proof. By assumption $(\chi)$ there holds, for $w>0, j=1, \ldots, N$ and $\mathbf{t} \in \mathbf{R}^{N}$,

$$
\begin{aligned}
\left|\left(K_{w, j} f\right)(\mathrm{t})-f(\mathrm{t})\right| & =\left|\sum_{\mathbf{k} \in \mathbf{Z}^{N}} w \int_{\frac{k_{j}}{w}}^{\frac{k_{j}+1}{w}} f\left(\frac{k_{1}}{w}, \ldots, u \ldots, \frac{k_{N}}{w}\right) d u \chi(w \mathrm{t}-\mathbf{k})-f(\mathrm{t})\right| \\
& =\left|\sum_{\mathbf{k} \in \mathbf{Z}^{N}}\left\{w \int_{\frac{k_{j}}{w}}^{\frac{k_{j}+1}{w}} f\left(\frac{k_{1}}{w}, \ldots, u \ldots, \frac{k_{N}}{w}\right) d u-f(\mathrm{t})\right\} \chi(w \mathrm{t}-\mathbf{k})\right| \\
& \leq \sum_{\mathbf{k} \in \mathbf{Z}^{N}}\left|w \int_{\frac{k_{j}}{w}}^{\frac{k_{j}+1}{w}} f\left(\frac{k_{1}}{w}, \ldots, u \ldots, \frac{k_{N}}{w}\right) d u-f(\mathrm{t})\right||\chi(w \mathbf{t}-\mathbf{k})| .
\end{aligned}
$$

Since $\chi$ has support contained in $[-T, T]^{N}, \chi(w \mathrm{t}-\mathrm{k})=0$ if $|w \mathrm{t}-\mathrm{k}|>T$, and therefore the series reduces to a finite sum over the indexes $\mathrm{k} \in \mathbf{Z}^{N}$ such that (wt - 
$\mathrm{k}) \in[-T, T]^{N}$, namely $\left|t_{i}-\frac{k_{i}}{w}\right| \leq \frac{T}{w}$, for every $i=1, \ldots, N$ : for such $\mathrm{k}$ we have that

$$
\left|w \int_{\frac{k_{j}}{w}}^{\frac{k_{j}+1}{w}} f\left(\frac{k_{1}}{w}, \ldots, u \ldots, \frac{k_{N}}{w}\right) d u-f(\mathrm{t})\right| \leq \omega_{1}\left(f ; \mathrm{t}, \frac{2 T}{w}, M\left(\mathbf{R}^{N}\right)\right),
$$

by the definition of the modulus of smoothness. Therefore

$$
\begin{aligned}
& \left|\left(K_{w, j} f\right)(\mathrm{t})-f(\mathrm{t})\right| \\
& \leq \sum_{(w \mathrm{t}-\mathrm{k}) \in[-T, T]^{N}}\left|w \int_{\frac{k_{j}}{w}}^{\frac{k_{j}+1}{w}} f\left(\frac{k_{1}}{w}, \ldots, u, \ldots, \frac{k_{N}}{w}\right) d u-f(\mathrm{t})\right||\chi(w \mathrm{t}-\mathbf{k})| \\
& \leq \omega_{1}\left(f ; \mathrm{t}, \frac{2 T}{w}, M\left(\mathbf{R}^{N}\right)\right) \sum_{(w \mathrm{t}-\mathrm{k}) \in[-T, T]^{N}}|\chi(w \mathrm{t}-\mathrm{k})| \\
& \leq A_{\chi} \omega_{1}\left(f ; \mathrm{t}, \frac{2 T}{w}, M\left(\mathbf{R}^{N}\right)\right),
\end{aligned}
$$

by $\left(\chi_{2}\right)$. Passing to the $L^{p}$-norm we obtain

$$
\left\|K_{w, j} f-f\right\|_{p} \leq A_{\chi} \tau_{1}\left(f ; \frac{2 T}{w}, M\left(\mathbf{R}^{N}\right)\right)_{p},
$$

and the thesis follows by Proposition 3.

\section{Convergence in variation for the multidimensional generalized sampling series}

We are now ready to prove the main result of the paper, that is, the convergence in variation for the multidimensional generalized sampling series with product kernels of averaged type. Since we will use results of the previous section, we assume here that $\chi$ is a kernel with compact support, that is, $\chi: \mathbf{R}^{N} \rightarrow \mathbf{R}$ satisfies assumption $(\chi)$.

Theorem 1. Let $f \in A C\left(\mathbf{R}^{N}\right)$ be such that $\frac{\partial f}{\partial x_{j}} \in \Lambda_{1} \cap R_{\mathrm{loc}}\left(\mathbf{R}^{N}\right)$, for every $j=1, \ldots, N$. Then, for every $m \in \mathbf{N}$,

$$
\lim _{w \rightarrow+\infty} V\left[\bar{S}_{w}^{m} f-f\right]=0 .
$$

Proof. Since $f \in A C\left(\mathbf{R}^{N}\right)$, by Proposition $1, \bar{S}_{w}^{m} f \in A C_{\mathrm{loc}}\left(\mathbf{R}^{N}\right)$, for every $w>0$ and $m \in \mathbf{N}$; therefore $\bar{S}_{w}^{m} f \in A C\left(\mathbf{R}^{N}\right)$ since $\bar{S}_{w}^{m} f \in B V\left(\mathbf{R}^{N}\right)$, as a consequence of Propositions 2 and 4 . Then, by Proposition 2,

$$
\begin{aligned}
V\left[\bar{S}_{w}^{m} f-f\right] & =\int_{\mathbf{R}^{N}}\left|\nabla \bar{S}_{w}^{m} f(\mathrm{t})-\nabla f(\mathrm{t})\right| d \mathrm{t} \\
& \leq \int_{\mathbf{R}^{N}} \sum_{j=1}^{N}\left|\frac{\partial}{\partial t_{j}}\left(\bar{S}_{w}^{m} f\right)(\mathrm{t})-\frac{\partial f}{\partial t_{j}}(\mathrm{t})\right| d \mathrm{t} \\
& =\sum_{j=1}^{N} \int_{\mathbf{R}^{N}}\left|\frac{1}{m} \sum_{i=1}^{m}\left(K_{j, w} \frac{\partial f}{\partial t_{j}}\right)\left(\mathrm{t}_{j}^{\prime}, t_{j}-\frac{m-2(i-1)}{2 w}\right)-\frac{\partial f}{\partial t_{j}}(\mathrm{t})\right| d \mathrm{t}
\end{aligned}
$$




$$
\begin{aligned}
\leq & \sum_{j=1}^{N} \frac{1}{m} \int_{\mathbf{R}^{N}} \mid \sum_{i=1}^{m}\left(K_{j, w} \frac{\partial f}{\partial t_{j}}\right)\left(\mathrm{t}_{j}^{\prime}, t_{j}-\frac{m-2(i-1)}{2 w}\right) \\
& -\sum_{i=1}^{m} \frac{\partial f}{\partial t_{j}}\left(\mathrm{t}_{j}^{\prime}, t_{j}-\frac{m-2(i-1)}{2 w}\right) \mid d \mathrm{t} \\
& +\sum_{j=1}^{N} \frac{1}{m} \int_{\mathbf{R}^{N}}\left|\sum_{i=1}^{m} \frac{\partial f}{\partial t_{j}}\left(\mathrm{t}_{j}^{\prime}, t_{j}-\frac{m-2(i-1)}{2 w}\right)-\frac{\partial f}{\partial t_{j}}(\mathrm{t})\right| d \mathrm{t} \\
\leq & \sum_{j=1}^{N} \| K_{w, j} \frac{\partial f}{\partial t_{j}}-\left.\frac{\partial f}{\partial t_{j}}\right|_{1} \\
& +\frac{1}{m} \sum_{j=1}^{N} \sum_{i=1}^{m} \int_{\mathbf{R}^{N}}\left|\frac{\partial f}{\partial t_{j}}\left(\mathrm{t}_{j}^{\prime}, t_{j}-\frac{m-2(i-1)}{2 w}\right)-\frac{\partial f}{\partial t_{j}}(\mathrm{t})\right| d \mathrm{t} \\
:= & \sum_{j=1}^{N} L_{j}+\frac{1}{m} \sum_{j=1}^{N} \sum_{i=1}^{m} I_{j}^{i} .
\end{aligned}
$$

Now, $L_{j} \rightarrow 0$ as $w \rightarrow+\infty$ by Proposition 4 , while $I_{j}^{i} \rightarrow 0$ as $w \rightarrow+\infty$, for every $i=1, \ldots, m, j=1, \ldots, N$, by the continuity in $L^{1}$ of the translation operator. Therefore the theorem is proved.

\section{Examples and applications to smoothing for digital image processing}

In this section, we will first prove a variation diminishing type property for the multidimensional generalized sampling series in case of step functions with compact support. We point out that this is exactly the case of functions that model digital images: we will then discuss some applications of such result to smoothing in digital image processing. Let us consider a step function $f: \mathbf{R}^{N} \rightarrow \mathbf{R}$ with compact support defined on a grid of intervals of length 1 , namely $f(\mathrm{x}):=\sum_{\mathrm{k} \in Z} a_{\mathrm{k}} \mathbf{1}_{\mathrm{k}}(\mathrm{x}), \mathrm{x} \in \mathbf{R}^{N}$, where $a_{\mathrm{k}} \in \mathbf{R}, Z=\left\{\left(\bar{k}_{1}+m_{1}, \ldots, \bar{k}_{N}+m_{N}\right), m_{i}=0,1, \ldots, l_{i}, i=1, \ldots, N\right\}$, for some $\overline{\mathrm{k}} \in \mathbf{Z}, l_{i} \in \mathbf{N}$, is a finite subset of $\mathbf{Z}$, and $\mathbf{1}_{\mathrm{k}}(\mathrm{x})$ is the characteristic function of the set $\prod_{i=1}^{N}\left[k_{i}, k_{i}+1\right), \mathrm{k} \in Z$, (i.e. $\mathbf{1}_{\mathrm{k}}(\mathrm{x})=1$, for $\mathrm{x} \in \prod_{i=1}^{N}\left[k_{i}, k_{i}+1\right)$ and $\mathbf{1}_{\mathrm{k}}(\mathrm{x})=0$ otherwise).

Proposition 5. Let $f: \mathbf{R}^{N} \rightarrow \mathbf{R}$ be a step function defined as above. Then, for every $w \in \mathbf{N}, m \in \mathbf{N}$,

$$
V\left[\bar{S}_{w}^{m} f\right] \leq N \prod_{i=1}^{N}\left\|\chi_{i}\right\|_{1} V[f] .
$$

Proof. Since obviously $f \in B V\left(\mathbf{R}^{N}\right)$, by Proposition 1 we have that $\bar{S}_{w}^{m} f \in$ $A C_{l o c}\left(\mathbf{R}^{N}\right)$ and $V\left[\bar{S}_{w}^{m} f\right]=\int_{\mathbf{R}^{N}}\left|\nabla\left(\bar{S}_{w}^{m} f\right)(\mathrm{t})\right| d \mathrm{t}$. For $j=1, \ldots, N$ and $\mathrm{t} \in \mathbf{R}^{N}$, 
by (3), there holds

$$
\begin{aligned}
\frac{\partial \bar{S}_{w}^{m} f}{\partial t_{j}}(\mathrm{t})= & \frac{w}{m} \sum_{\mathbf{k} \in \mathbf{Z}^{N}} f\left(\frac{\mathbf{k}}{w}\right) \prod_{i \neq j} \bar{\chi}_{i, m}\left(w t_{i}-k_{i}\right) \\
& \cdot\left[\chi_{j}\left(w t_{j}-k_{j}+\frac{m}{2}\right)-\chi_{j}\left(w t_{j}-k_{j}-\frac{m}{2}\right)\right] \\
= & \frac{w}{m} \sum_{\mathbf{k} \in \mathbf{Z}^{N}} f\left(\frac{\mathbf{k}}{w}\right) \prod_{i \neq j} \bar{\chi}_{i, m}\left(w t_{i}-k_{i}\right) \cdot \chi_{j}\left(w t_{j}-k_{j}+\frac{m}{2}\right) \\
- & \frac{w}{m} \sum_{\widetilde{\mathbf{k}} \in \mathbf{Z}^{N}} f\left(\frac{\widetilde{\mathbf{k}}_{j}^{\prime}}{w}, \frac{\widetilde{k}_{j}-m}{w}\right) \prod_{i \neq j} \bar{\chi}_{i, m}\left(w t_{i}-\widetilde{k}_{i}\right) \cdot \chi_{j}\left(w t_{j}-\widetilde{k}_{j}+\frac{m}{2}\right) \\
= & \frac{w}{m} \sum_{\mathbf{k} \in \mathbf{Z}^{N}}\left[f\left(\frac{\mathbf{k}}{w}\right)-f\left(\frac{\mathbf{k}_{j}^{\prime}}{w}, \frac{k_{j}-m}{w}\right)\right] \prod_{i \neq j} \bar{\chi}_{i, m}\left(w t_{i}-k_{i}\right) \cdot \chi_{j}\left(w t_{j}-k_{j}+\frac{m}{2}\right),
\end{aligned}
$$

where, in the second series, we have put $\widetilde{k}_{i}=k_{i}$ for $i \neq j$ and $\widetilde{k}_{j}=k_{j}+m$. Therefore

$$
\begin{aligned}
V\left[\bar{S}_{w}^{m} f\right] & =\int_{\mathbf{R}^{N}}\left|\nabla\left(\bar{S}_{w}^{m} f\right)(\mathrm{t})\right| d \mathrm{t} \leq \int_{\mathbf{R}^{N}} \sum_{j=1}^{N}\left|\frac{\partial\left(\bar{S}_{w}^{m} f\right)(\mathrm{t})}{\partial t_{j}}\right| d \mathrm{t} \\
& \leq \int_{\mathbf{R}^{N}} \sum_{j=1}^{N}\left[\frac{w}{m} \sum_{\mathbf{k} \in \mathbf{Z}^{N}}\left|f\left(\frac{\mathbf{k}_{j}^{\prime}}{w}, \frac{k_{j}}{w}\right)-f\left(\frac{\mathbf{k}_{j}^{\prime}}{w}, \frac{k_{j}-m}{w}\right)\right| \prod_{i \neq j}\left|\bar{\chi}_{i, m}\left(w t_{i}-k_{i}\right)\right|\right. \\
& \left.\cdot\left|\chi_{j}\left(w t_{j}-k_{j}+\frac{m}{2}\right)\right|\right] d \mathrm{t} \\
& =\sum_{j=1}^{N}\left[\frac{w}{m} \sum_{\mathbf{k} \in \mathbf{Z}^{N}}\left|f\left(\frac{\mathbf{k}_{j}^{\prime}}{w}, \frac{k_{j}}{w}\right)-f\left(\frac{\mathbf{k}_{j}^{\prime}}{w}, \frac{k_{j}-m}{w}\right)\right| \int_{\mathbf{R}^{N}} \prod_{i \neq j}\left|\bar{\chi}_{i, m}\left(w t_{i}-k_{i}\right)\right|\right. \\
& \left.\cdot\left|\chi_{j}\left(w t_{j}-k_{j}+\frac{m}{2}\right)\right| d \mathrm{t}\right] .
\end{aligned}
$$

If we now put $u_{j}=w t_{j}-k_{j}+\frac{m}{2}$ and $u_{i}=w t_{i}-k_{i}$, for $i \neq j$,

$$
\begin{aligned}
V\left[\bar{S}_{w}^{m} f\right] & \leq \frac{1}{m w^{N-1}} \sum_{j=1}^{N} \sum_{\mathbf{k} \in \mathbf{Z}^{N}} V_{\left[\frac{k_{j}-m}{w}, \frac{k_{j}}{w}\right]}\left[f\left(\frac{\mathbf{k}_{j}^{\prime}}{w}, \cdot\right)\right] \prod_{i \neq j}\left\|\bar{\chi}_{i, m}\right\|_{1}\left\|_{\chi_{j}}\right\|_{1} \\
& \leq \frac{1}{m w^{N-1}} \prod_{i=1}^{N}\left\|\chi_{i}\right\|_{1} \sum_{j=1}^{N} \sum_{\mathbf{k} \in \mathbf{Z}^{N}} V_{\left[\frac{k_{j}-m}{w}, \frac{k_{j}}{w}\right]}\left[f\left(\frac{\mathbf{k}_{j}^{\prime}}{w}, \cdot\right)\right] \\
& \leq \frac{1}{m w^{N-1}} \prod_{i=1}^{N}\left\|\chi_{i}\right\|_{1} \sum_{j=1}^{N} \sum_{\mathbf{k} \in \mathbf{Z}^{N}}\left[V_{\left[\frac{k_{j}-m}{w}, \frac{k_{j}-m+1}{w}\right]}\left[f\left(\frac{\mathbf{k}_{j}^{\prime}}{w}, \cdot\right)\right]+\ldots\right. \\
& \left.+V_{\left[\frac{k_{j}-1}{w}, \frac{k_{j}}{w}\right]}\left[f\left(\frac{\mathbf{k}_{j}^{\prime}}{w}, \cdot\right)\right]\right] \\
& =\frac{1}{w^{N-1}} \prod_{i=1}^{N}\left\|\chi_{i}\right\|_{1} \sum_{j=1}^{N} \sum_{\mathbf{k}_{j}^{\prime} \in \mathbf{Z}^{N-1}} V_{\mathbf{R}}\left[f\left(\frac{\mathbf{k}_{j}^{\prime}}{w}, \cdot\right)\right]
\end{aligned}
$$


Now, if $B=\prod_{i=1}^{N}\left[p_{i}, q_{i}\right]$ denotes the support of $f$,

$$
\begin{aligned}
V\left[\bar{S}_{w}^{m} f\right] & \leq \frac{1}{w^{N-1}} \prod_{i=1}^{N}\left\|\chi_{i}\right\|_{1} \sum_{j=1}^{N} \sum_{\mathrm{k}_{j}^{\prime} \in \mathbf{Z}^{N-1}} V_{\left[p_{j}, q_{j}\right]}\left[f\left(\frac{\mathrm{k}_{j}^{\prime}}{w}, \cdot\right)\right] \\
& =\prod_{i=1}^{N}\left\|\chi_{i}\right\|_{1} \sum_{j=1}^{N} \sum_{\mathrm{k}_{j}^{\prime} \in \mathbf{Z}^{N-1}} \int_{\left[\frac{\mathrm{k}_{j}^{\prime}}{w}, \frac{\mathrm{k}_{j}^{\prime}+1}{w}\right]} V_{\left[p_{j}, q_{j}\right]}\left[f\left(\frac{\mathrm{k}_{j}^{\prime}}{w}, \cdot\right)\right] d \mathrm{u}_{j}^{\prime} \\
& =\prod_{i=1}^{N}\left\|\chi_{i}\right\|_{1} \sum_{j=1}^{N} \int_{\left[\mathrm{p}_{j}^{\prime}, \mathrm{q}_{j}^{\prime}\right]} V_{\left[p_{j}, q_{j}\right]}\left[f\left(\mathrm{u}_{j}^{\prime}, \cdot\right)\right] d \mathrm{u}_{j}^{\prime}=\prod_{i=1}^{N}\left\|\chi_{i}\right\|_{1} \sum_{j=1}^{N} \Phi_{j}(f, B),
\end{aligned}
$$

where we have used the fact that $f$ is constant on each interval $\left[\frac{\mathrm{k}_{j}^{\prime}}{w}, \frac{\mathrm{k}_{j}^{\prime}+1}{w}\right]$. Therefore, since of course $\Phi_{j}(f, B) \leq V_{B}[f]$, for every $j=1, \ldots, N$, we conclude that

$$
V\left[\bar{S}_{w}^{m} f\right] \leq N \prod_{i=1}^{N}\left\|\chi_{i}\right\|_{1} V[f] .
$$

We point out that, in case of non-negative kernels, the previous result gives a classical variation diminishing type property, being $\left\|\chi_{i}\right\|_{1}=1$ for every $i=1, \ldots, N$. Moreover such result, in the one-dimensional setting $(N=1)$, is a particular case of Proposition 1 of [6], given for a general $B V$-function, where the estimate must be as follows

$$
V\left[\bar{S}_{w}^{m} f\right] \leq\|\chi\|_{1} V[f]
$$

(i.e., in Proposition 1 of $[6]$ the constant that multiplies $\|\chi\|_{1} V[f]$ should be 1 ) for $f \in B V(\mathbf{R}), w>0, m \in \mathbf{N}$.

We now provide some basic examples of kernels for which the above results hold, and we discuss some applications of the variation diminishing type property (5) to smoothing in digital image processing.

As stated in Section 2, in this paper we consider product kernels $\bar{\chi}_{m}$ of averaged type.

As a first example, we can consider the multivariate product kernel of the averaged type generated by the well-known Fejér kernel (see, e.g., Figure 1 left and [27]), defined by:

$$
F(x):=\frac{1}{2} \operatorname{sinc}^{2}(x / 2), \quad x \in \mathbf{R},
$$

where the sinc-function (see, e.g., $[26,7])$ is of the form:

$$
\operatorname{sinc}(x):= \begin{cases}\sin (\pi x) / \pi x, & x \neq 0 \\ 1, & x=0\end{cases}
$$



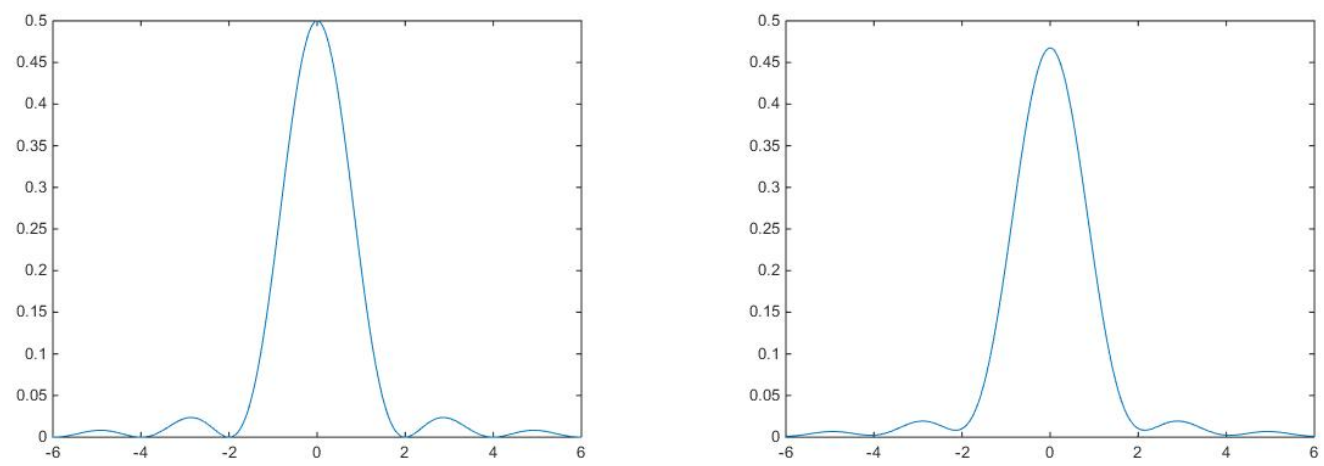

Figure 1. On the left: the Fejér kernel $F$. On the right: the averaged Fejér kernel with $m=1$.

Now, the one-dimensional averaged Fejér kernel (see, e.g., Figure 1, right) is the following:

$$
\bar{F}_{m}(t):=\frac{1}{2 m} \int_{-m / 2}^{m / 2} \operatorname{sinc}^{2}\left(\frac{t+v}{2}\right) d v, \quad t \in \mathbf{R}, \quad m \in \mathbf{N}
$$

and the corresponding multivariate version (see, e.g., Figure 2 for the case $N=2$ ) is:

$$
\mathcal{F}_{m}(\mathrm{t}):=\prod_{i=1}^{N} \bar{F}_{m}\left(t_{i}\right), \quad \mathrm{t} \in \mathbf{R}^{N} .
$$

It is well-known that the Fejér kernel has unbounded support and satisfies assumptions $\left(\chi_{1}\right)$ and $\left(\chi_{2}\right)$ (see e.g., $\left.[31,8]\right)$, then for the multivariate generalized sampling series based upon $\mathcal{F}_{m}$ holds the variation diminishing type property established in Proposition 5, when suitable multivariate step-type signals are considered.

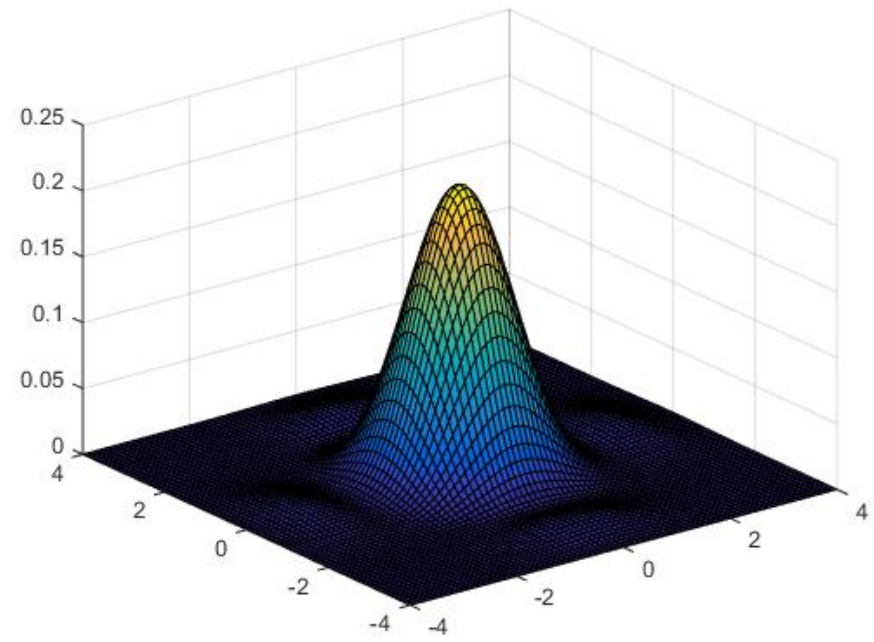

Figure 2. The bivariate averaged Fejér kernel with $m=1$.

Moreover, also the relation established in Proposition 2 holds, where the $j$-th first order partial derivatives of the generalized sampling series of a given absolutely continuous function $f$ is related with $K_{w, j} \frac{\partial f}{\partial t_{j}}$, i.e., the corresponding sampling series of the Kantorovich type of the $j$-th first order partial derivatives of $f$. 
However, the convergence results proved in Section 4 can not be applied to the generalized sampling series based upon $\mathcal{F}_{m}$ since the kernels $\mathcal{F}_{m}$ do not have compact support, hence assumption $(\chi)$ is not satisfied.

Other examples of one-dimensional kernels with unbounded support that can be used to define product averaged type kernels can be found, e.g., in [38, 28, 29, 30].

In order to recall examples of kernels of one variable such that the corresponding multivariate averaged type versions also satisfy assumption $(\chi)$, we recall the definition of the well-known central B-spline of order $n \in \mathbf{N}$ (see, e.g., Figure 3 and $[46,25,2,3,20])$, defined by:

$$
M_{n}(x):=\frac{1}{(n-1) !} \sum_{i=0}^{n}(-1)^{i}\left(\begin{array}{c}
n \\
i
\end{array}\right)\left(\frac{n}{2}+x-i\right)_{+}^{n-1}, \quad x \in \mathbf{R},
$$

where $(x)_{+}:=\max \{x, 0\}$ denotes "the positive part" of $x \in \mathbf{R}$ (see e.g., [41, 32]). The functions $M_{n}(x)$ are non-negative, continuous with compact support contained in $[-n / 2, n / 2]$, and satisfy conditions $\left(\chi_{1}\right)$ and $\left(\chi_{2}\right)$.

Now, let us denote by

$$
\bar{M}_{n, m}(t):=m^{-1} \int_{-m / 2}^{m / 2} M_{n}(t+v) d v, \quad t \in \mathbf{R},
$$

the averaged B-spline kernel of order $n \in \mathbf{N}$. Recalling the following well-known property:

$$
M_{n}^{\prime}(t)=M_{n-1}(t+1 / 2)-M_{n-1}(t-1 / 2), \quad t \in \mathbf{R}, \quad(n \geq 2)
$$

for $m=1$, we have:

$$
\bar{M}_{n, 1}^{\prime}(t)=M_{n}(t+1 / 2)-M_{n}(t-1 / 2)=M_{n+1}^{\prime}(t), \quad t \in \mathbf{R}, \quad(n \geq 1),
$$

i.e., $\bar{M}_{n, 1}(t)=M_{n+1}(t)+k, k \in \mathbf{R}$. Now, since $\bar{M}_{n, 1}$ belongs to $L^{1}(\mathbf{R})$ (see [6]), we must have $k=0$ and therefore we conclude that

$$
\bar{M}_{n, 1}(t)=M_{n+1}(t), \quad t \in \mathbf{R},
$$

for every $n \in \mathbf{N}$, namely, the averaged kernel with $m=1$ generated by a central $\mathrm{B}$-spline of order $n$ is a B-spline itself of order $n+1$.
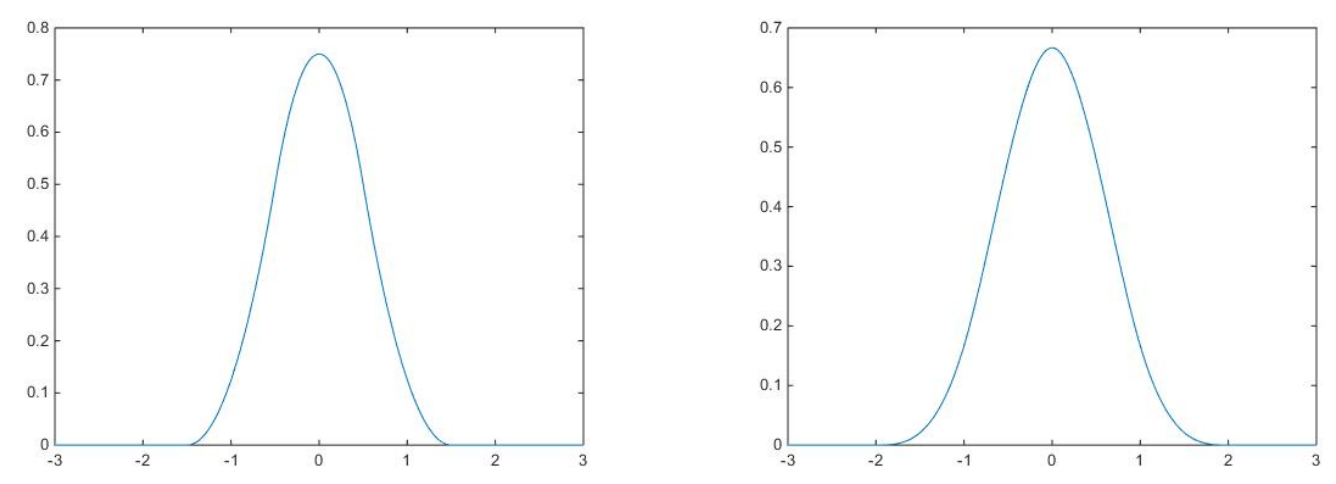

Figure 3. On the left: the central B-spline $M_{2}$. On the right: the central B-spline $M_{3}$ which corresponds to $\bar{M}_{2,1}$.

In view of the above remark, we can explicitly state that the multivariate averaged type kernel with $m=1$ and generated by $M_{n}$ (see, e.g., Figure 4 for the case $n=2$ 
in two space dimension) is the following:

$$
\mathcal{M}_{1}^{n}(\mathrm{t}):=\prod_{i=1}^{N} \bar{M}_{n, 1}\left(t_{i}\right)=\prod_{i=1}^{N} M_{n+1}\left(t_{i}\right), \quad \mathrm{t} \in \mathbf{R}^{N} .
$$

In practice, in the latter case the multivariate generalized sampling operators based upon averaged B-spline $M_{n}$ with $m=1$ coincide with the usual generalized sampling series based upon the multivariate central B-spline of order $n+1$.

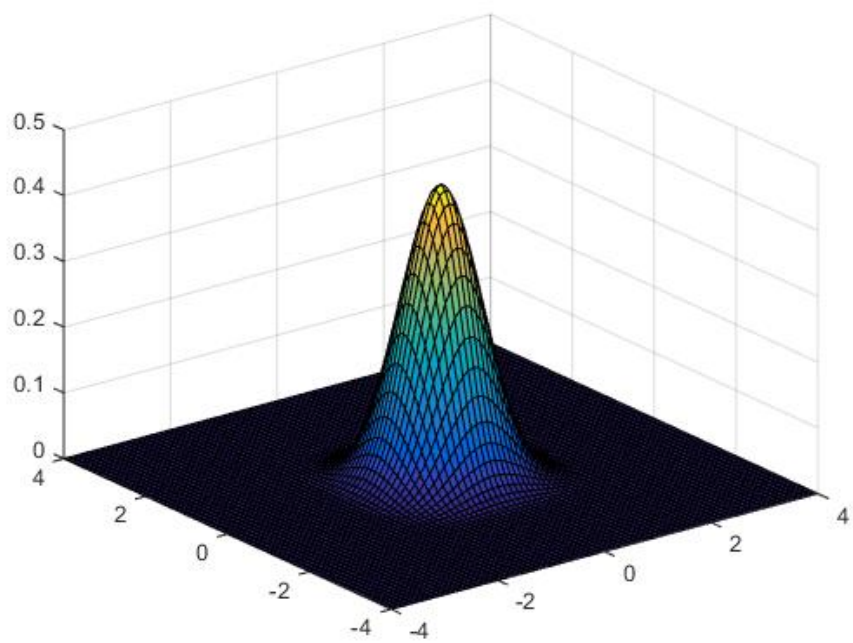

Figure 4. The bivariate averaged B-spline kernel of order 2 with $m=1$ which coincides with $\mathcal{M}_{1}^{2}(\mathrm{t})=\prod_{i=1}^{2} M_{3}\left(t_{i}\right)$

Generally speaking, we can also define the multivariate average central B-spline kernels as follows:

$$
\mathcal{M}_{m}^{n}(\mathrm{t}):=\prod_{i=1}^{N} \bar{M}_{n, m}\left(t_{i}\right)=m^{-1} \prod_{i=1}^{N} \int_{-m / 2}^{m / 2} M_{n}\left(t_{i}+v\right) d v, \quad \mathrm{t} \in \mathbf{R}^{N} .
$$

Since $\mathcal{M}_{m}^{n}$ has compact support it satisfies also assumption $(\chi)$; thus the above sampling series fulfills the results of both Section 3 and Section 5. Further, for the latter examples of kernels also Proposition 4 and Theorem 1 can be applied.

Now, at the end of this section we consider some applications to smoothing in digital processing. For basic facts concerning this numerical tool for imaging, see e.g., [19, 39, 43].

It is well-known that any static gray scale image is a bivariate signal with compact support; then it can be (naturally) modeled as follows:

$$
I_{A}(x, y):=\sum_{i=1}^{m} \sum_{j=1}^{m} a_{i j} \cdot \mathbf{1}_{i j}(x, y) \quad\left((x, y) \in \mathbf{R}^{2}\right),
$$

for every image (matrix) $A=\left(a_{i j}\right)_{i j}, i, j=1,2, \ldots, m$, where $\mathbf{1}_{i j}(x, y), i, j=$ $1,2, \ldots, m$, is the characteristic function of the sets $[i-1, i) \times[j-1, j)$ (i.e. $\mathbf{1}_{i j}(x, y)=1$, for $(x, y) \in[i-1, i) \times[j-1, j)$ and $\mathbf{1}_{i j}(x, y)=0$ otherwise $)$.

Note that the above function $I_{A}(x, y)$ is defined in such a way that to every pixel $(i, j)$ it is associated the corresponding gray level $a_{i j}$. 
Moreover, by the above representation $I_{A}$ of the image $A$ it turns out that $I_{A} \in B V\left(\mathbf{R}^{2}\right)$, hence one can consider approximations of $A$ by means of the bivariate generalized sampling series based upon the averaged type kernels. The main advantage that can be achieved by the above procedure is expressed by Proposition 5 , that can be applied to $I_{A}$ : the variation of $\bar{S}_{w}^{m} I_{A}$ is controlled by the variation of $I_{A}$ and, following the proof of Proposition 5, in case of non-negative kernels, we obtain that the "Tonelli integrals" of $\bar{S}_{w}^{m} I_{A}$ are smaller than those ones of $I_{A}$, $\left(\Phi_{j}\left(\bar{S}_{w}^{m} I_{A}, B\right) \leq \Phi_{j}\left(I_{A}, B\right), j=1, \ldots, N\right.$, where $B$ is the support of $\left.f\right)$ i.e., in some sense, the operators provide a regularized approximated version of the original image A.

Clearly, in order to visualize an approximation (new image) of the original image $A$ by means of $\bar{S}_{w}^{m} I_{A}$, we need to sample the operators, for $w \in \mathbf{N}$, with a fixed sampling grid. Obviously, the sampling grid is chosen arbitrarily hence one can also consider different (high) sampling grids.

The effect of the proposed procedure can be strongly noticed at the edges of the figures, where the jumps of gray levels are reduced with respect to the corresponding ones in the original image.

Now we can give the following practical examples of image reconstruction in order to show the smoothing capabilities of the above operators. An optimized version of the above described algorithm for image reconstruction and smoothing can be implemented by means of the MATLAB programming language, following the indications outlined in $[14,15]$ in case of the so-called sampling Kantorovich algorithm for image enhancement.

For the numerical experiments, we consider the well-known images of Lena and Baboon with $150 \times 150$ pixel resolution (see Figure 5).
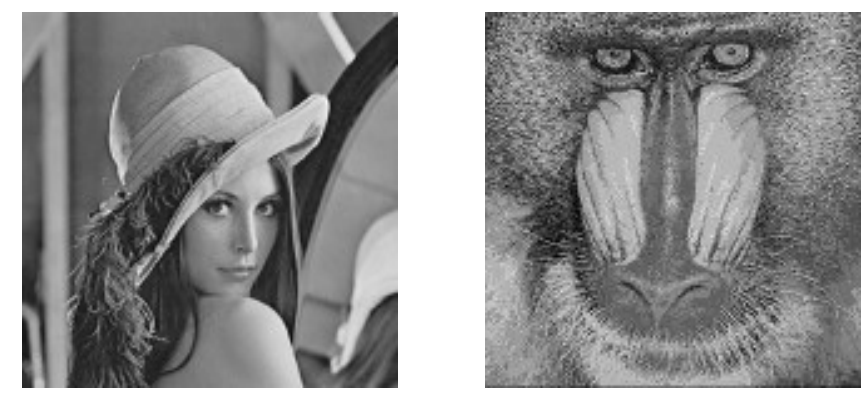

Figure 5. On the left: Lena. On the right: Baboon.
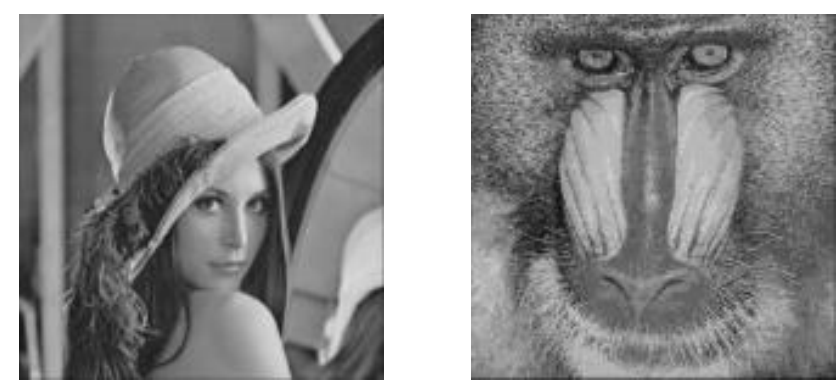

Figure 6 . On the left: the reconstruction of Lena (of $150 \times 150$ pixel) by means of the operator $\bar{S}_{w}^{m}, w=4$, based upon the bivariate averaged Fejér kernel with $m=4$. On the right: the reconstruction of Baboon made as for Lena.

First of all, we reconstruct the original images in Figure 5 by using the bivariate averaged Fejér kernel with $m=4$. As observed above, following the proof of 
Proposition 5 and taking into account that $\|F\|_{1}=1$, it is possible to show that

$$
\Phi_{j}\left(\bar{S}_{w}^{4} f, B\right) \leq \Phi_{j}(f, B),
$$

for every $j=1, \ldots, N$ (being $B$ the support of $f$ ), i.e., the Tonelli integrals (that are related to the variation of the sections of the function) of the reconstructed image are smaller than those ones of the original image, therefore producing the smoothing effect. In Figure 6 we have the reconstruction of Lena and Baboon (of $150 \times 150$ pixel resolution) by means of the operator $\bar{S}_{w}^{m}, w=4$, based upon the bivariate averaged Fejér kernel with $m=4$. By detailed analysis of the edges (especially in case of Lena, at the contours of the hat) it is possible to observe the smoothing of the analyzed images.
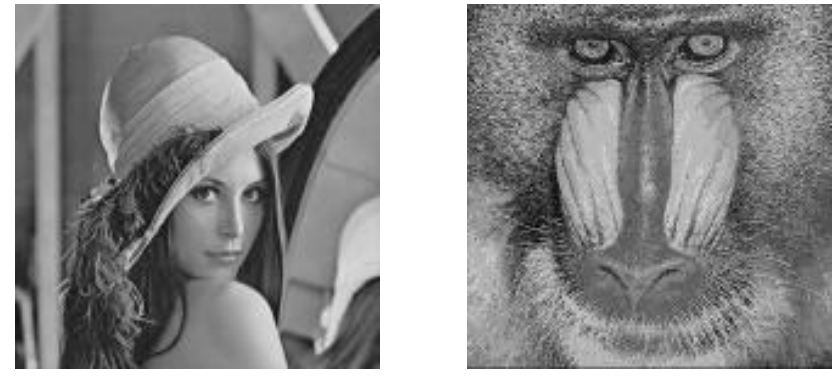

Figure 7. On the left: the reconstruction of Lena (of $150 \times 150$ pixel) by means of the operator $\bar{S}_{w}^{m}, w=10$, based upon the bivariate averaged Fejér kernel with $m=4$. On the right: the reconstruction of Baboon made as for Lena.

In Figure 7 we have the reconstruction of Lena and Baboon (of $150 \times 150$ pixel resolution) by means of the operator $\bar{S}_{w}^{m}, w=10$, based upon the bivariate averaged Fejér kernel with $m=4$.

The main differences that can be observed between the images in Figure 6 and Figure 7 are that, for big values of $w$ the images are closer to the original and consequently the edges tend to be more clear.
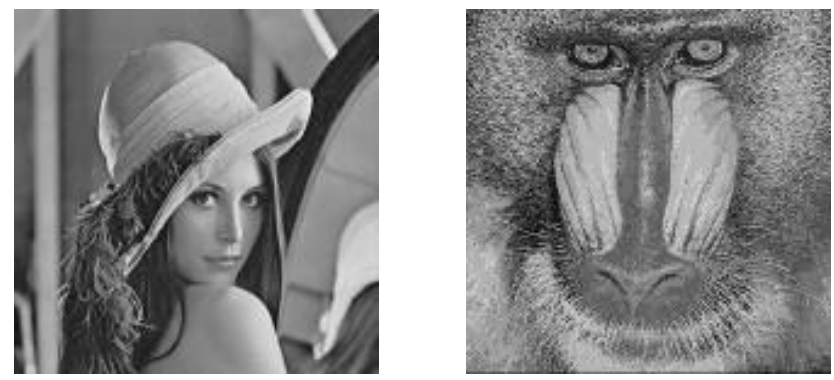

Figure 8. On the left: the reconstruction of Lena (of $150 \times 150$ pixel) by means of the operator $\bar{S}_{w}^{m}, w=6$, based upon the bivariate averaged central B-spline of order 3 with $m=4$. On the right: the reconstruction of Baboon made as for Lena.

Finally, in Figure 8 we have the reconstruction of Lena and Baboon (of $150 \times 150$ pixel resolution) by means of the operator $\bar{S}_{w}^{m}, w=6$, based upon the bivariate central B-spline of order 3 with $m=4$. Note that, also for the latter case one can state a inequality as that given in (7) since also $\left\|M_{3}\right\|_{1}=1$.

Acknowledgments. The authors are members of the Gruppo Nazionale per l'Analisi Matematica, la Probabilitá e le loro Applicazioni (GNAMPA) of the Istituto Nazionale di Alta Matematica (INdAM). 
The authors are partially supported by the "Department of Mathematics and Computer Science" of the University of Perugia (Italy). The first and the third author have been partially supported within the projects "Metodi di teoria degli operatori e di Analisi Reale per problemi di approssimazione ed applicazioni", "Metodi di Teoria dell'Approssimazione, Analisi Reale, Analisi Nonlineare e loro applicazioni" and "Integrazione, Approssimazione, Analisi Nonlineare e loro Applicazioni", funded by the 2017, 2018 and 2019 basic research fund of the University of Perugia. Finally, the first and the second author of the paper have been partially supported within a 2019 GNAMPA-INdAM Project: "Metodi di analisi reale per l'approssimazione attraverso operatori discreti e applicazioni".

\section{References}

[1] Agratini, O.: An approximation process of Kantorovich type. - Math. Notes Miskolc 2:1, 2001, 3-10.

[2] Allasia, G., R. Cavoretto, and A. De Rossi: A class of spline functions for landmarkbased image registration. - Math. Methods Appl. Sci. 35, 2012, 923-934.

[3] Allasia, G., R. Cavoretto, and A. De Rossi: Lobachevsky spline functions and interpolation to scattered data. - Comput. Appl. Math. 32, 2013, 71-87.

[4] Ambrosio, L., and S. Di Marino: Equivalent definitions of BV space and of total variation on metric measure spaces. - J. Funct. Anal. 266:7, 2014, 4150-4188.

[5] Angeloni, L.: Approximation results with respect to multidimensional $\varphi$-variation for nonlinear integral operators. - Z. Anal. Anwend. 32:1, 2013, 103-128.

[6] Angeloni, L., D. Costarelli, and G. Vinti: A characterization of the convergence in variation for the generalized sampling series. - Ann. Acad. Sci. Fenn. Math. 43, 2018, 755-767.

[7] Angeloni, L., D. Costarelli, and G. Vinti: A characterization of the absolute continuity in terms of convergence in variation for the sampling Kantorovich operators. - Mediterr. J. Math. 16, 2019, 44.

[8] Angeloni, L., D. Costarelli, and G. Vinti: Quantitative estimates for sampling type operators with respect to the Jordan variation. - Atti Accad. Naz. Lincei Cl. Sci. Fis. Mat. Natur. Rend. Lincei (9) Mat. Appl., 2020 (to appear).

[9] Angeloni, L., and G. Vinti: Convergence and rate of approximation for linear integral operators in $B V^{\varphi}$-spaces in multidimensional setting. - J. Math. Anal. Appl. 349, 2009, 317334.

[10] Angeloni, L., and G. Vinti: Approximation in variation by homothetic operators in multidimensional setting. - Differential Integral Equation 26:5-6, 2013, 655-674.

[11] Angeloni, L., and G. Vinti: Convergence and rate of approximation in $B V^{\varphi}\left(\mathbf{R}_{+}^{N}\right)$ for a class of Mellin integral operators. - Atti Accad. Naz. Lincei Cl. Sci. Fis. Mat. Natur. Rend. Lincei (9) Mat. Appl. 25:3, 2014, 217-232.

[12] Angeloni, L., and G. Vinti: Convergence in variation and a characterization of the absolute continuity. - Integral Transforms Spec. Funct. 26:10, 2015, 829-844.

[13] Appell, J., J. Banaś, and N. Merentes: Bounded variation and around. - de Gruyter Ser. Nonlinear Anal. Appl. 17, De Gruyter, Berlin, Germany, 2014.

[14] Asdrubali, F., G. Baldinelli, F. Bianchi, D. Costarelli, A. Rotili, M. Seracini, and G. Vinti: Detection of thermal bridges from thermographic images by means of image processing approximation algorithms. - Appl. Math. Comput. 317, 2018, 160-171.

[15] Asdrubali, F., G. Baldinelli, F. Bianchi, D. Costarelli, L. Evangelisti, A. Rotili, M. SERAcini, and G. Vinti: A model for the improvement of thermal bridges quantitative assessment by infrared thermography. - Applied Energy 211, 2018, 854-864. 
[16] Bardaro, C., P. L. Butzer, R. L. Stens, and G. Vinti: Prediction by samples from the past with error estimates covering discontinuous signals. - IEEE Trans. Inform. Theory 56:1, $2010,614-633$.

[17] Bardaro, C., I. Mantellini, R. Stens, J. Vautz, and G. Vinti: Generalized sampling approximation for multivariate discontinuous signals and application to image processing. - New Perspectives on Approximation and Sampling Theory-Festschrift in honor of Paul Butzer's 85th birthday, Birkhauser, 2014, 87-114.

[18] Bardaro, C., and G. Vinti: A general approach to the convergence theorems of generalized sampling series. - Appl. Anal. 64, 1997, 203-217.

[19] BI, S., X. HAN, and Y. YU: An $L_{1}$ image transform for edge-preserving smoothing and scene-level intrinsic decomposition. - ACM Transactions on Graphics 34:4, 2015, Art. no. 78.

[20] Bozzini, M., L. Lenarduzzi, M. Rossini, and R. Schaback: Interpolation with variably scaled kernels. - IMA J. Numer. Anal. 35:1, 2015, 199-219.

[21] Butzer, P. L., A. Fisher, and R. L. Stens: Generalized sampling approximation of multivariate signals: theory and applications. - Note Mat. 10:1, 1990, 173-191.

[22] Butzer, P. L., A. Fisher, and R. L. Stens: Generalized sampling approximation of multivariate signals: general theory. - Atti Sem. Mat. Fis. Univ. Modena 41:1, 1993, 17-37.

[23] Butzer, P. L., and R. J. Nessel: Fourier analysis and approximation I. - Academic Press, New York-London, 1971.

[24] Butzer, P. L., W. Splettstösser, and R. L. Stens: The sampling theorem and linear prediction. - Jahresber. Deutsch. Math.-Verein. 90, 1988, 1-70.

[25] Charina, M., C. Conti, K. Jetter, and G. Zimmermann: Scalar multivariate subdivision schemes and box splines. - Computer Aided Geometric Design 28:5, 2011, 285-306.

[26] Coroianu, L., and S. G. GaL: $L^{p}$-approximation by truncated max-product sampling operators of Kantorovich-type based on Fejer kernel. - J. Integral Equations Appl. 29:2, 2017, 349-364.

[27] Costarelli, D., A. M. Minotti, and G. Vinti: Approximation of discontinuous signals by sampling Kantorovich series. - J. Math. Anal. Appl. 450:2, 2017, 1083-1103.

[28] Costarelli, D., and G. Vinti: Approximation by max-product neural network operators of Kantorovich type. - Results Math. 69:3, 2016, 505-519.

[29] Costarelli, D., and G. Vinti: Max-product neural network and quasi-interpolation operators activated by sigmoidal functions. - J. Approx. Theory 209, 2016, 1-22.

[30] Costarelli, D., and G. Vinti: Pointwise and uniform approximation by multivariate neural network operators of the max-product type. - Neural Networks 81, 2016, 81-90.

[31] Costarelli, D., and G. Vinti: An inverse result of approximation by sampling Kantorovich series. - Proc. Edinb. Math. Soc. (2) 62:1, 2019, 265-280.

[32] D'Amore, L., R. Campagna, A. Galletti, L. Marcellino, and A. Murli: A smoothing spline that approximates Laplace transform functions only known on measurements on the real axis. - Inverse Problems 28:2, 2012, 025007.

[33] Dolženko, E. P., and E. A. Sevast'Janov: Approximations of functions in the Hausdorff metric by means of piecewise monotone (in particular, rational) functions. - Mat. Sb. (N.S.) 101(143):4, 1976, 508-541 (in Russian).

[34] Korovkin, P. P.: Attempt at an axiomatic construction in certain problems in the theory of approximation of functions of one variable. - Kalinin. Gos. Ped. Inst. Učen. Zap. 69, 1969, 91-109 (in Russian).

[35] Giusti, E.: Minimal surfaces and functions of bounded variation. - Monographs in Mathematics 80, Birkhäuser Verlag, Basel, 1984.

[36] Gousseau, Y., and J. M. Morel: Are natural images of bounded variation? SIAM J. Math. Anal. 33:3, 2001, 634-648. 
[37] Mantellini, I., and G. Vinti: Approximation results for nonlinear integral operators in modular spaces and applications. - Ann. Polon. Math. 81:1, 2003, 55-71.

[38] Orlova, O., and G. TAmberG: On approximation properties of generalized Kantorovich-type sampling operators. - J. Approx. Theory 201, 2016, 73-86.

[39] PAn, J., X. YAng, H. CAI, and B. Mu: Image noise smoothing using a modified Kalman filter. - Neurocomputing 173:3, 2016, 1625-1629

[40] Radó, T.: Length and area. - Amer. Math. Soc. Colloq. Publ. 30, Amer. Math. Soc., New York, 1948.

[41] Romani, L., and M. A. SABIn: The conversion matrix between uniform B-spline and Bézier representations. - Computer Aided Geometric Design 21:6, 2004, 549-560.

[42] Sendov, B., and V.A. Popov: The averaged moduli of smoothness. - Wiley, Chichester, U.K., 1988.

[43] Sonka, M., V. Hlavac, and R. Boyle: Image processing, analysis, and machine vision. Cengage Learning, Australia - Brazil - Japan, 2014.

[44] Strong, D., and T. Chan: Edge-preserving and scale-dependent properties of total variation regularization. - Inverse Problems 19:6, 2003.

[45] Tonelli, L.: Su alcuni concetti dell'analisi moderna. - Ann. Scuola Norm. Super. Pisa. (2) 11, 1942, 107-118.

[46] Unser, M.: Ten good reasons for using spline wavelets. - Wavelets Applications in Signal and Image Processing 3169:5, 1997, 422-431.

[47] Valkonen, T., K. Bredies, and F. Knoll: Total generalized variation in diffusion tensor imaging. - SIAM J. Imaging Sci. 6:1, 2013, 487-525.

[48] Vinti, C.: Perimetro-variazione. - Ann. Scuola Norm. Sup. Pisa (3) 18, 1964, 201-231. 